\section{Neue Beweglichkeit durch Technik?}

\author{
Bereits seit Beginn der Forschung über Telearbeit wurde damit argumentiert, \\ daß die Nutzung neuer Formen der Telekommunikation im Sinne eines Telekom- \\ munikation-Transport-Tradeoffs auch das Bewegungsverhalten im Raum verän- \\ dert. Welche Trends sind real zu erwarten?
}

I

Von Rainer König

m Zeitalter der Telekommunikation werden

vielfach Substitutions- und Induktionseffekte und Veränderungen in der Struktur der Bewegungsaktivitäten erwartet. Unter Verweis auf die bislang weitgehend parallele Entwicklung von technisch vermittelten Fernkommunikationsund Verkehrssystemen wird einerseits grundsätzlich die Frage aufgeworfen, inwieweit nicht die Idee, Verkehr durch Kommunikation zu ersetzen, längst ausgeschöpft ist (1). Andererseits wird der Telekommunikation in jüngster Zeit eine zentrale Rolle bei der Lösung der Verkehrsproblematik zugemessen (2).

Zahlreiche Pilotprojekte jüngeren Datums wie außerbetriebliche Arbeitsstätten trugen dazu bei, praktische Erfahrungen über Anwendungen der Telekommunikation zur Substitution physischen Verkehrs zu sammeln. Empirisch gesicherte Angaben über die Verkehrssubstitution wie beispielsweise durch Videokonferenzen anstelle der Geschäftsreise liegen bisher aber nur sehr lïckenhaft und sind in der Tendenz keineswegs einheitlich (3). Die Beurteilung von Richtung und Ausmaß der Wirkungen wird zusätzlich erschwert durch die enge Verschränktheit von Verkehrs- und Kommunikationsnetzen mit gesellschaftlichen Teilsystemen. Sie wirken quasi als Katalysator der vorherrschenden Rahmenbedingungen. Einige Indizien erlauben dennoch eine grobe Einordnung der verkehrlichen Wirkungen von Telekommunikationsanwendungen und unterstreichen die vermuteten Ambivalenzen (siehe Abbildung) Informations- und Kommunikationssysteme sind einerseits wesentliche Triebkräfte für den wirtschaftlichen Strukturwandel, in dem sie neue Formen der Arbeitsteilung beziehungsweise der räumlichen Ausdehnung von Beschaffungs- und Absatzstrategien ermöglichen (z.B. global sourcing, just-in-time) und damit strukturelle Wachstumsimpulse im Verkehr erzeugen. Neue Formen der Telekommunikation können aber auch in bestimmten Verkehrssegmenten einen ,verkehrseinsparenden" Charak- ter haben. Beispiele hierfür sind die ReduzieGeschäftsreisen. In der wissenschaftlichen Diskussion wird mehrheitlich davon ausgegangen, daß die verkehrsinduzierende Effekte per Saldo überwiegen. Eine differenzierte Betrachtung möglicher verkehrssparender Anwendungen ist dennoch angebracht.

\section{- Pendller auf der Datenautobahn}

Das Erdbeben in Los Angeles 1994, nach dem es vielen Arbeitnehmern unmöglich war, ihren Arbeitsplatz zu erreichen und nur wenige mit ihren Arbeitgebern über Datenleitung vom heimischen Computer aus Verbindung aufnehmen konnten, wirkte als auslösendes Moment für eine breitere Diskussion. Auch die zahlreichen Initiativen und Pilotprojekte zum Thema „Multimedia“ oder "Information Superhighway“ haben diesen Ansatz aufgegriffen. Telekommunikationsanwendungen zur Verkehrssubstitution werden unter anderem in der Telearbeit zur Vermeidung von Berufsverkehr, im Bildtelefon bzw. in Telekonferenzen (Telekooperation, Joint Editing) zur Vermeidung von Geschäfts- und Dienstreiseverkehr, im Teleshopping zur Vermeidung von Einkaufsverkehr sowie im Teleunterricht zur Vermeidung von Ausbildungsverkehr gesehen.

Betrachtet man beispielsweise Telearbeit, so entstanden erste Konzepte bereits in den achtziger Jahren und waren geprägt durch die Erfahrungen des ersten Ölpreisschocks 1973 und durch das Bewußtsein der Krise der Industriegesellschaft. Die Einrichtung von Satellitenbüros führte zu beträchtlichen Einsparungen für Unternehmen und Beschäftigte. Im Einklang mit anderen am technisch Machbaren orientierten Leitbildern (z.B. „papierloses Büro“, „menschenleere Fabrik") ermutigte dies Prognostiker zur Schätzung von bis zu 40 Prozent Substitutionspotential im Berufsverkehr. Dabei wurde eine wesentlich schnellere Diffusion informations- und kommunikationstechnischer Anwenrung von Leerfahrten oder der Ersatz von dungen unterstellt, als diese tatsächlich eintrat. Individuelle, organisatorische und soziale Rahmenbedingungen, die eine wesentliche Voraussetzung für die erfolgreiche Einführung neuer Technologien darstellen, wurden dagegen stark unterschätzt.

\section{Zwei Panel-Studien}

Zwei Panel-Studien aus den USA bzw. aus den Niederlanden, in denen das Verkehrsverhalten durch teilnehmende Beobachtung bei Versuchsteilnehmern und einer Kontrollgruppe ermittelt wurde, sind besonders interessant. In der USamerikanischen Studie, in der Arbeitnehmer durchschnittlich an 1,5 Tagen pro Woche Teleworking nutzten, konnte eine signifikante Verringerung der Fahrtenhäufigkeit (bis zu 50 Prozent) und der zuriickgelegten Strecken (20 Prozent) festgestellt werden. Auch nichtberufliche Aktivitäten der Teleworker wurden näher an den Wohnort verlagert. Teleworking führte entgegen den Erwartungen nicht zu einem Ansteigen von Freizeitverkehr und sogar die anderen Haushaltsmitglieder wurden zu einer effizienteren Verkehrsteilnahme angeregt. Auch in der niederländischen Studie konnten vergleichbare Ergebnisse ermittelt werden, wenngleich die Verringerung der Fahrtenhäufigkeit mit 17 Prozent deutlich geringer ausfiel, dennoch erreichte die Âbnahme zu Hauptverkehrszeiten mit 25 Prozent beachtliche Ausmaße. Auf den gesamtstädtischen Verkehr hochgerechnet, würde sich dieser damit um bis zu fünf Prozent verringern lassen. Bei der Interpretation dieser Ergebnisse, insbesondere bei einer Übertragung der amerikanischen Zahlen auf europäische Verhältnisse spielen zwei Aspekte eine wesentliche Rolle. Zum einen besteht mit dem "Clean Air Act" in Kalifornien eine politische Zielvorgabe an Unternehmen, das Aufkommen im Berufsverkehr bei den Beschäftigten zu reduzieren, zum anderen sind die durchschnittlichen Entfernungen zwischen Arbeitsplatz und Wohnung in den USA länger als in Europa.

In der Gesamtschau der vorliegenden Untersuchungen und Erfahrungen aus den einzelnen Anwendungsbereichen wird deutlich, daß grundsätzlich ein Potential besteht, physischen Verkehr durch Telekommunikation zu ersetzen. Es wird aber auch deutlich, daß vorrangig an technologischen Leitbildern orientierte optimistische Schätzungen aus der Vergangenheit teilweise erheblich nach unten korrigiert werden mußten. Zehn bis 20 Prozent Substitutionspotential 


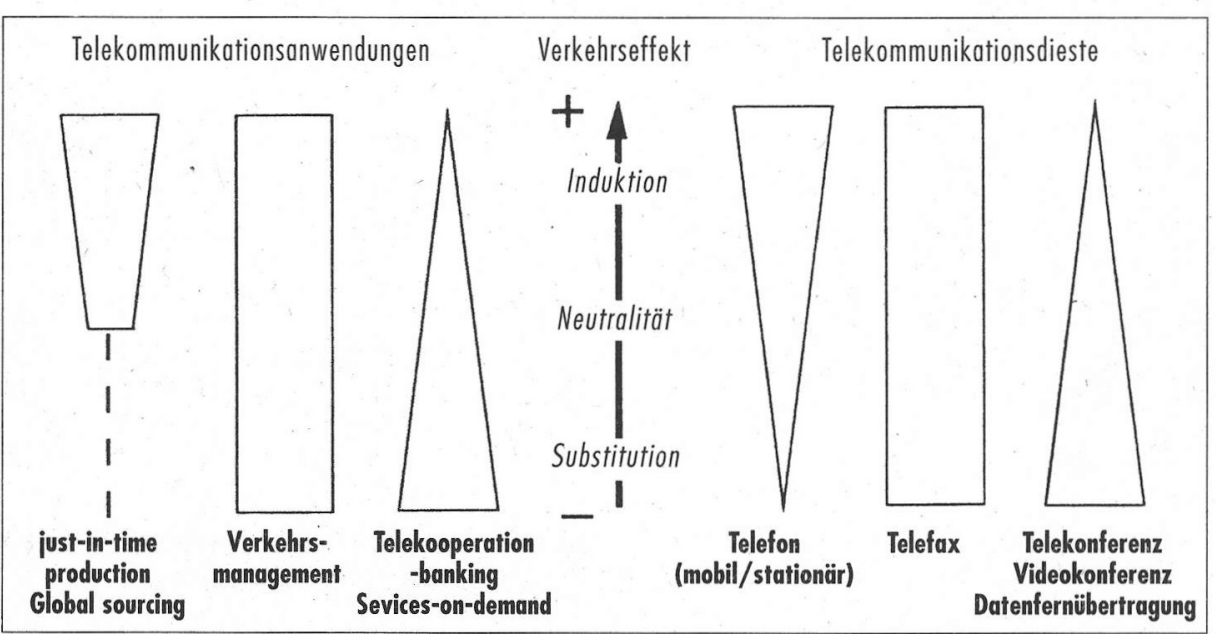

Quelle: Fraunhofer-|SI, Deutsche Telekom

der Verkehrsleistung für bestimmte Fahrtzwecke werden in der Regel nicht überschritten. Läßt man nun die auf den Freizeitverkehr bezogenen Anwendungen - die bislang nur in Visionen zum Ausdruck kommen - außen vor, und berücksichtigt man den Stellenwert der unterschiedlichen Fahrtzwecke am motorisierten Individualverkehr insgesamt, so beläuft sich das gesamte Einsparpotential auf rund acht Prozent der Personenverkehrsleistung. Die größten Beiträge hierzu liefern Geschäfts-/Dienstreiseverkehr mit 3,5 Prozent und Berufsverkehr mit 2,9 Prozent.

\section{Rahmenbedingungen und Gestaltungsansätze}

Angesichts struktureller Wachstumsimpulse der Telekommunikation auf den Verkehr besteht damit zumindest die Hoffnung auf einen Ausgleich der Wachstumsraten im Verkehr. Erwartungen zu einer weitergehenden Lösung von Verkehrsproblemen werden durch die vorliegenden Untersuchungen nicht untermauert. Denn aus ihnen geht hervor, daß die Ausschöpfung der vorhandenen Substitutionspotentiale an zahlreiche er-folgsbestimmende Rahmenbedingungen gebunden ist. Mit Blick auf die vorherrschenden - eher angebotsorientierten - Strategien in Forschung und Entwicklung impliziert dies eine Abkehr von technologischen Leitbildern hin zu nutzerorientierten Anwendungskonzepten, die Aktivierung von Gestaltungspotentialen infolge der wirtschaftlichen und gesellschaftlichen Dynamik sowie die Notwendigkeit verkehrspolitischer Gesamtstrategien:

- Statt neue Pilotprojekte zu starten, sollten Probleme und Ergebnisse der bisherigen Anwendungen und Pilotprojekte zum Ausgangspunkt zukünftiger Projekte für eine umfassende Gestaltung der Technik gemacht werden.
- Da Akzeptanzbarrieren bei der Nutzung neuer Mehrwertdienste meist auf fehlende Anwendungskonzepte und Implementierungsstrategien zurückzuführen sind, sollten Einflußfaktoren der Adoption und Nutzung der Telekommunikation stärker als bisher in die verkehrliche Diskussion miteinbezogen werden.

- Die Substitution kann nur vorankommen, wenn die Telekommunikationssysteme nutzerzentriert entwickelt und gestaltet werden, so daß sie ihrerseits positive Gefühle und Erlebnisse vermitteln. Unbedingt notwendig ist also eine entsprechende psychologische Forschung.

- Zur Stimulierung der Nachfrage nach neuen Diensteangeboten sind auch neue Wege in der Tarifgestaltung zu betreten.

- Inwieweit die Verteuerung des Individualverkehrs zu einer verstärkten Telekommunikationsnutzung führt, ist bisher nicht empirisch erforscht worden und sollte deshalb untersucht werden.

- Da Verkehrsvermeidung aufgrund spezifischer Kalküle der Akteure meist als nichtintendierter Nebeneffekt der Nutzung von Telekommunikation auftritt, ist zu untersuchen, inwieweit Implementations- und Nutzungsstrategien aus den USA, die gezielt zur Verkehrsvermeidung eingesetzt werden, auch auf Europa übertragen werden können.

- Bei den Wechselwirkungen der Effekte des Telekommunikationseinsatzes und der Veränderung der Rahmenbedingungen gibt es noch einen umfangreichen Bedarf an Forschung.

- Die öffentliche Diskussion um die Verkehrsvermeidung einschließlich der Substitution durch Telekommunikation muß gefördert werden. Dazu müssen die verschiedenen Akteure im Hinblick auf eine verkehrspolitische Gesamtstrategie koordiniert werden.
- Die mit dem gesellschaftlichen und wirtschaftlichen Wandel verbundenen Innovationsprozesse bergen ein erhöhtes Ge-staltungspotential auch für die Entwicklung und Einführung verkehrsvermeidender Organisationsformen in sich. Im Zuge dieser Innovationsprozesse muß ein Diskurs über alternative Formen der Mobilität in Gang gesetzt werden, der die Diffusion und Nutzung von Telekommunikation miteinbezieht.

- Die gesellschaftlichen und wirtschaftlichen Wirkungen des Telekommunikationseinsatzes sollten verstärkt in der verkehrswissenschaftlichen Diskussion berïcksichtigt werden; hier ist noch ein erheblicher Forschungsbedarf zu verzeichnen.

- Von der zunehmenden Dysfunktionalität des Verkehrssystems werden in Zukunft mit Sicherheit die stärksten Impulse für eine verbesserte Auslastung der Infrastrukturkapazitäten ausgehen. Beim Einsatz von Telekommunikation zur Verkehrssteuerung sollten Strategien zur Verkehrsvermeidung durch Telematik stets mit eingeschlossen sein und entwickelt werden.

- Um erfolgreich Konzepte zur Verkehrsvermeidung zu entwickeln, muß vor allem die bisher stark segmentierte Betrachtungsweise der Verkehrsforschung sowie der Telekommunikationsforschung aufgehoben werden.

\section{Literatur}

1) H. Lübbe: Mobilität - vorerst unaufhaltsam. in: Internationales Verkehrswesen 45, 11, S. 653-658, 1993

2) A.B. Boghani, E.W. Kimble, E.E. Spencer: Can Telecommunications Help Solve America's Transportation Problems? A Multiclient Study. Cambridge, Mass.: Arthur D. Little; 1991

3) S. Köhler: Interdependenzen zwischen Telekommunikation und Personenverkehr. Schriftenreihe des Instituts für Städtbau und Landesplanung der Universität Fridericiana zu Karlsruhe, Heft 24, Karlsruhe: Universität, 1993

- D.-M. Harmsen, R. König: Möglichkeiten der Substitution physischen Verkehrs durch Telekommunikation. Abschlubbericht für das Forschungs- und Technologiezentrum der Deutschen Telekom, Darmstadt, Karlsruhe: FhG-ISI, Juli 1994

\section{Der Autor}

Rainer König ist Dipl. Wirtschaftsingenieur, Leiter der Forschungsgruppe Marktentwicklungspotential und sozio-ökonomisches Umfeld, Deutsche Telekom AG, Technologiezentrum Darmstadt

Kontakt: Rainer König, Jollysir. 39, 76137

Karlsruhe, Tel. (07-21)-83-74-30, Fax -29 
(c) 20I0 Authors; licensee IÖW and oekom verlag. This is an article distributed under the terms of the Creative Commons Attribution Non-Commercial No Derivates License (http://creativecommons.org/licenses/by-nc-nd/3.o/), which permits unrestricted use, distribution, and reproduction in any medium, provided the original work is properly cited. 